\title{
Cloud Enhancement of Global Horizontal Irradiance in California and Hawaii
}

\author{
Rich H. Inman, Yinghao Chu, Carlos F. M. Coimbra* \\ Department of Mechanical and Aerospace Engineering, Jacobs School of Engineering \\ Center for Renewable Resource Integration and Center for Energy Research \\ University of California San Diego, La Jolla, CA 93093, USA
}

\section{Abstract}

Clouds significantly attenuate ground-level solar irradiance causing substantial reduction in photovoltaic power output capacity. However, partly cloudy skies may lead to temporary enhancement of local Global Horizontal Irradiance (GHI) above the clearsky ceiling and, at times, the extraterrestrial irradiance. Such enhancements are referred to here as Cloud Enhancement Events (CEEs). In this work we study these CEEs and assess quantitatively the occurrence of resulting coherent Ramp Rates (RRs). We analyze a full year of ground irradiance data recorded at the University of California, Merced, as well as nearly five months of irradiance data recorded at the University of California, San Diego, and Ewa Beach, Hawaii. Our analysis shows that approximately $4 \%$ of all the data points qualify as potential CEEs, which corresponds to nearly 3.5 full-days of such events per year if considered sequentially. The surplus irradiance enhancements range from $18 \mathrm{~W} \mathrm{~m}^{-2} \mathrm{day}^{-1}$ to $73 \mathrm{~W} \mathrm{~m}^{-2} \mathrm{day}^{-} 1$. The maximum recorded GHI of $\sim 1,400 \mathrm{~W} \mathrm{~m}^{-2}$ occurred in San Diego on May 25, 2012, which was nearly 43\% higher than the modeled clear-sky ceiling. Wavelet decomposition coupled with fluctuation power index analysis shed light on the time-scales on which cloud induced variability and CEEs operate. Results suggest that while cloud-fields tend to induce variability most strongly at the 30 minute time-scale, they have the potential to cause CEEs that induce variability on time-scales of several minutes. This analysis clearly demonstrates that CEEs are an indicator for periods of high variability and therefore provide useful information for solar forecasting and integration. Keywords: Global Horizontal Irradiance; Cloud Enhancement; Total Sky Imager.

\section{Introduction}

The total extraterrestrial beam irradiance incident on the earth's atmosphere $I_{0}$ fluctuates about an average value of approximately $1,360 \mathrm{~W} \mathrm{~m}^{-2}$ (Kopp and Lean, 2011). This inci- ${ }^{13}$ dent radiation is attenuated as it negotiates its way to ground ${ }^{14}$ level through a complex series of multiple reflections, absorp- ${ }^{15}$ tions and re-emissions due to interactions with atmospheric con- ${ }^{16}$ stituents (Goody and Yung, 1995). This results in the divi- ${ }^{17}$ sion of the incident extraterrestrial beam radiation into two dis- ${ }^{18}$ tinct components; Direct Normal Irradiance (DNI) and Diffuse ${ }^{19}$ Horizontal Irradiance (DHI), the geometric sum of which is ${ }^{20}$

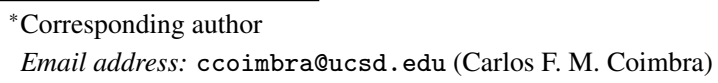

Preprint submitted to Solar Energy equation,

$$
\mathrm{GHI}=\mathrm{DNI} \cos \theta_{z}+\mathrm{DHI}
$$

where $\theta_{z}$ is the solar zenith angle, see Figure 1

In addition to the partitioning of the radiation, atmospheric cloud-formation is typically associated with a pronounced decrease in the intensity of solar irradiance components. In fact, the attenuation of incoming solar radiation by clouds is routinely larger than any other atmospheric component (Inman et al. 2013). Furthermore, the driving effects of clouds on radiative energy budgets include short wave cooling, as a result of absorption of incoming solar radiation, and long wave heating, 


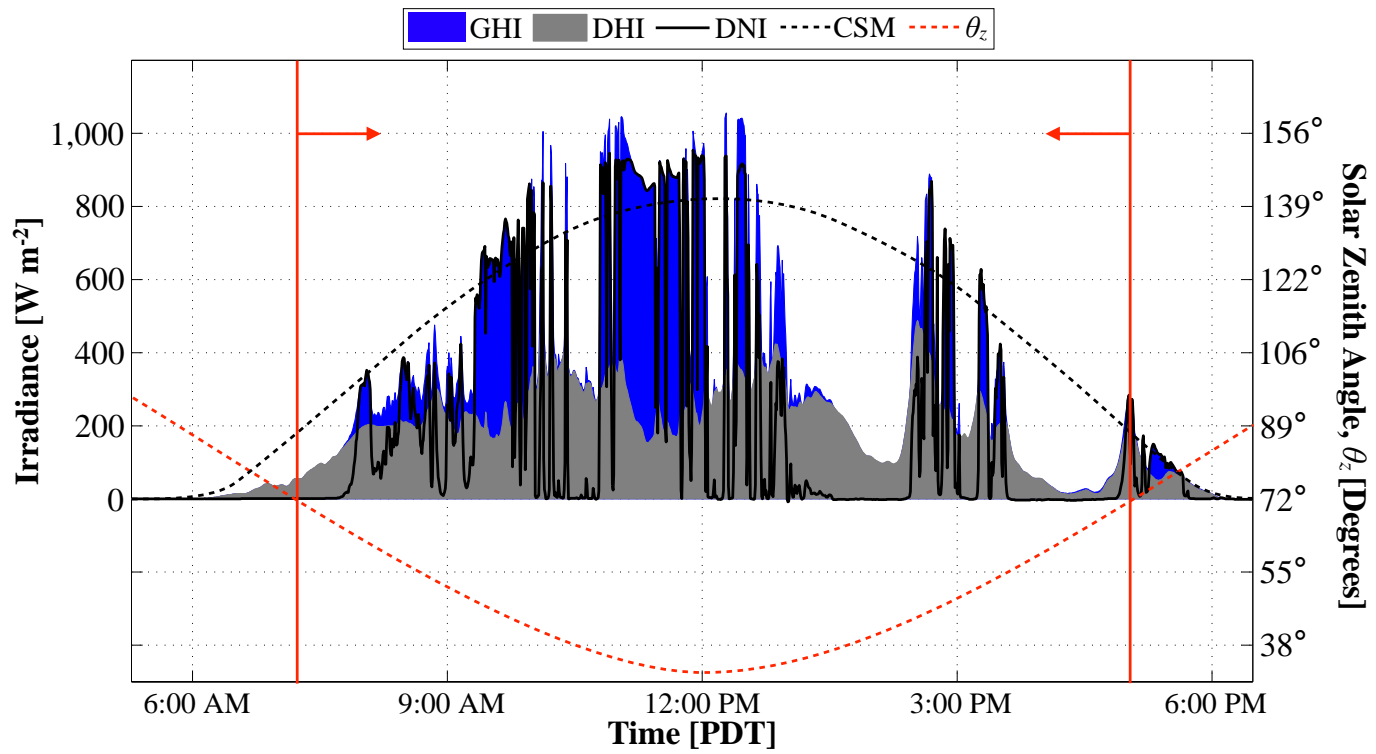

Figure 1: Components of solar irradiance sampled every 30 seconds at the University of California, Merced, on March 21, 2011. Global Horizontal Irradiance (GHI) was measured with a Precision Spectral Pyranometer (PSP), manufactured by the Eppley Laboratory, Inc. Direct Normal Irradiance (DNI) was measured using a Normal Incidence Pyrheliometer (NIP) and 2-axis automatic solar tracker (SMT-3), both of which are also available from the Eppley Laboratory, Inc. Diffuse Horizontal Irradiance (DHI) was measured using an additional PSP and SMT-3 with accompanying Shade Disk Kit (SDK). Solar zenith angle is also plotted and observations with $\cos \theta_{z} \leq 0.3\left(\theta_{z} \geq 72.5^{\circ}\right)$, shown outside solid red-lines, being excluded from the study. The Clear-Sky Model (CSM) used in this figure is explained in detail in $\$ 3.2$

may lead to the reverse; i.e., multiple scatterings and reflections 43 of short wave radiation by cloud fields may lead to increased ${ }_{44}$ irradiance from a portion of the sky above the corresponding 45 cloud-free value (Franceschini, 1968; Wen et al., 2001; Wyser ${ }_{46}$ et al., 2002; Pfister et al., 2003, Emck and Richter, 2008; Berg $_{47}$ et al. 2011). In rare occasions, these enhancements can cause 48 the local GHI to instantaneously exceed the extraterrestrial so- 49 lar constant $I_{0}$ (McCormick and Suehrcke, 1990; Tapakis and ${ }_{50}$ Charalambides, 2014). Such enhancement of GHI above the 51 corresponding clear-sky value are referred to here as Cloud En- 52 hancement Events (CEEs).

With the exception of two studies, one by Luoma et al. 54 (2012) and a second by Tapakis and Charalambides (2014), 55 little work has been done in the realm of CEEs with respect 56 to PhotoVoltaic (PV) power generation. In this work we do 57 not intend to suggest a new mechanism by which CEEs occur, 58 but rather investigate the coherent Ramp Rates (RRs) associ- 59 ated with CEEs and their potential impact on the quality of PV 60 power generation. To clarify, a coherent CEE RR is defined as 61 a series of monotonically increasing or decreasing GHI observations whose maximum value exceeds the expected clear-sky value by a given threshold. These CEEs and their associated RRs are of interest for several reasons: current models typically do not consider the ability of clouds to increase the local available irradiance, these events commonly precede or follow periods of lower than normal irradiance associated with the presence of passing opaque clouds leading to relatively large RRs, large RRs can cause voltage flicker that in turn trigger tap chargers on distribution feeders increasing operations cost for utilities, and therefore the successful forecasting of these events could lead to an effective control scheme to reduce the cost associated with high levels of variability in photovoltaic power generation.

Analysis of the amplitude, persistence, and frequency occurrence of ground-level irradiance fluctuation requires a decomposition of the input time-series into a set of orthogonal sub-signals each representing a specified time-scale of fluctuation. Due to the stochastic and non-periodic nature of the atmo- 
spheric processes that drive ground-level fluctuations in clear-100 ness, Fourier analysis is typically not suitable. Alternatively, 101 spectral analysis of high frequency (e.g., $1 \mathrm{~s}-1 \mathrm{~min}$ ) irradiance ${ }_{102}$ time-series on a wavelet basis rather than a periodic basis can be ${ }_{103}$ found in the literature. Kawasaki et al. (2006) decomposed 2-104 years of 1-min irradiance data from 9 sites in a $4 \times 4 \mathrm{~km}$ grid using the Daubechies 4 wavelet (Hazewinkel, 2001). Woyte et al.05 (2007) applied the Haar (1911) wavelet to clearness index timeseries and defined a fluctuation power index (fpi) that quantified the amplitude and frequency occurrence of variability on speci- ${ }^{107}$ fied time-scales. Perpiñán and Lorenzo (2011) analyzed several ${ }^{108}$ days of $1 \mathrm{~s}$ irradiance time-series using the MOD-WT wavelet ${ }^{109}$ and later used wavelet transform correlations to study fluctua- ${ }^{110}$ tions of the electrical power generated by an ensemble of $70^{111}$ DC/AC inverters from a 45.6 MW PV plant (Perpiñán et al. ${ }^{112}$ 2013). Lave et al. (2012) applied a wavelet transform to clear- ${ }^{113}$ sky index time-series from a single site to the average of $\operatorname{six}^{114}$ sites and showed a strong reduction in variability at short time- ${ }^{115}$ scales (i.e., shorter than 5-min) with lesser reductions at longer ${ }^{116}$ time-scales. Lave et al. (2013) also developed a wavelet-based ${ }^{117}$ variability model for PV plant output and Lave et al. (2013) em- ${ }^{118}$ ployed the wavelet-based model to study the impact of cloud ${ }^{119}$ speed on solar variability scaling. Similarly, Peled and Appel- ${ }^{20}$ baum (2013) used statistical tools and wavelet analysis to de- ${ }^{12}$ velop estimators for the magnitude of voltage and power varia ${ }^{122}$ tions within PV systems due to climatic conditions.

The time-scales of these events have been shown to range ${ }^{124}$ from seconds (Schade et al. 2007), to 15-30 mins Segal and Davis, 1992, Tapakis and Charalambides, 2014), and occasionally as long as hours (Cede et al. 2002). Here we employ a wavelet decomposition using the top-hat wavelet to show that while the CEE RRs typically only last a few minutes they are generally correlated with variability on longer time-scales associated with the passing of cloud fields.

This study makes use of a full year of irradiance data recorded at the University of California, Merced, as well as nearly five months of irradiance data recorded at the University of California, San Diego, and Ewa Beach, Hawaii, see $\$ 2$ Methods are described in $\$ 3$ which includes descriptions of the clear-sky model, statistical analysis, ramp rate calculation, wavelet decomposition, and fluctuation power index analysis. The results of these analyses are covered in $\$ 4$ and the conclusions in $\$ 5$ respectively.

\section{Data}

\subsection{Irradiance Data}

Irradiance data are collected at three locations characterized by different micro-climates: Merced California (continental), San Diego California (coastal), and Ewa Beach Hawaii (island). A broadband (285 - 2,800 nm) Eppley Precision Spectral Pyranometer (PSP) and Normal Incidence Pyrheliometer (NIP) are used in Merced (Mar. 21, 2011 to Jun. 19, 2012) to collect GHI and DNI data, respectively. Two Multi-Filter Rotating Shadowband Radiometers (Model MFR-7), available from Yankee Environmental Systems (YES), which measure both GHI and DNI are used to collect irradiance data in San Diego and Ewa Beach during the period from Jan. 27, 2012 to Jun. 17, 2012. Irradiance data are logged using Campbell Scientific CR-1000 data-loggers at a sampling rate of 30 seconds. It is important to note that due to the 30 second sampling rate used in this study the observed RRs are likely lower than what would be observed „with an increased sampling rate, see for example Yordanov et al. (2013)a, Yordanov et al. (2013)b, and Almeida et al. (2014).

\subsection{Selection of $G H I$}

This work focuses on GHI rather than DNI due to the nature of the cloud enhancement process. CEEs are necessarily observable in the diffuse component of irradiance and manifest in the global component of irradiance as a result of Eq. (1). This is because CEEs result from clouds elevating short wave diffuse irradiance above that of the corresponding clear-sky value with little or no change in DNI, which is illustrated through an analysis of DNI clear-sky indices,

$$
k_{D}(t)=\frac{\mathrm{DNI}(t)}{\mathrm{DNI}_{\mathrm{clr}}(t)}
$$




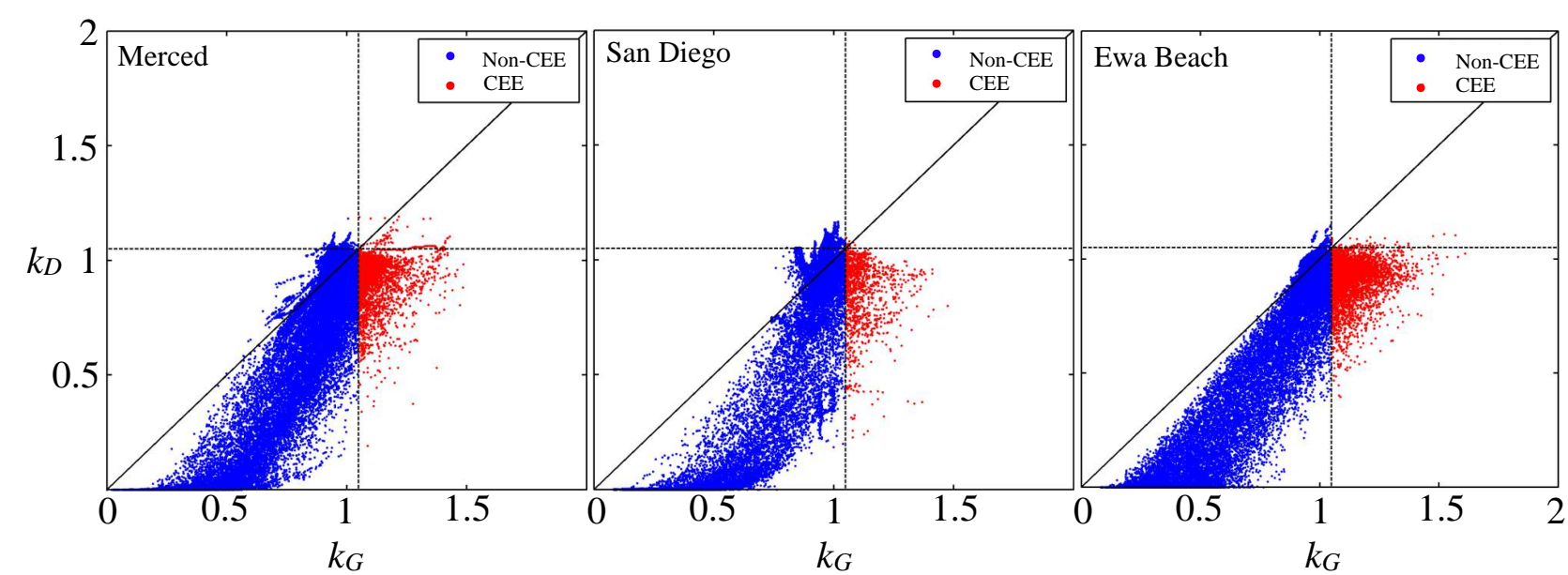

Figure 2: Scatter plots of $k_{D}$ versus $k_{G}$ for irradiance measurements in Merced, CA (left); San Diego, CA (center); and Ewa Beach, HI (right). Each marker stands for a single observation. Red markers represent GHI CEE cases and the dash lines represent the 1.05 threshold for both $k_{D}$ and $k_{G}$.

Table 1: Correlation coefficients between DNI clear-sky indices and GHI clear- ${ }^{138}$ sky indices for overall, non-CEE, and CEE periods at different locations.

\begin{tabular}{llll}
\hline & \multicolumn{3}{c}{ Correlation coefficient } \\
\hline & Overall & Non-CEE & CEE \\
\hline Merced & 0.9 & 0.92 & 0.07 \\
San Diego & 0.9 & 0.91 & -0.16 \\
Ewa Beach & 0.94 & 0.95 & -0.03 \\
\hline
\end{tabular}

and GHI clear-sky indices

$$
k_{G}(t)=\frac{\mathrm{GHI}(t)}{\mathrm{GHI}_{\mathrm{clr}}(t)}
$$

where $\mathrm{GHI}_{\mathrm{clr}}(t)$ and $\mathrm{DNI}_{\mathrm{clr}}(t)$ are generated from the Clear-Sky Model (CSM) described in $\$ 3.2$ As shown in Figure 2 $k_{D}{ }^{149}$ generally increases with $k_{G}$ for non-CEE periods and typically ${ }^{150}$ results in correlation coefficients greater than 0.9 , which is to be expected (see Table 1). On the other hand, during CEE periods, the correlation between $k_{D}$ and $k_{G}$ is low and in some cases ${ }^{151}$ negative (see Table 1). It is also clear that during CEE periods, ${ }^{152}$ $k_{G}$ is typically in the range of $1.05-1.5$ while $k_{D}$ tends to occupy ${ }^{153}$ the range from $0.5-1.0$. It is also important to note that there ${ }^{154}$ are some occasions where $k_{D}$ did exceed 1.05 , however, such ${ }^{155}$ enhancement was found to result from errors in the DNI CSM ${ }^{156}$ at elevated zenith angles under cloudless skies and, as a result, ${ }^{157}$ were excluded from the study.

\section{Methods}

\subsection{Data Quality}

Data from the early morning and late afternoon are excluded from the analysis. There are several motivations for their removal: primarily, the cosine response of pyranometer measurements are typically maximized for $\theta_{z} \gtrsim 70^{\circ}$; secondly, times when $\theta_{z} \gtrsim 70^{\circ}$ are associated with a relatively high airmass resulting in a large fraction of GHI originating from the diffuse component; and finally, the high solar zenith angle in combination with an elevated airmass results in rather low photovoltaic power production. To this end, a threshold is applied to the solar zenith angle $\theta_{z}$ according to,

$$
\cos \theta_{z} \geq 0.3
$$

with data not satisfying Equation (4), which corresponds to $\theta_{z} \geq$ $72.5^{\circ}$, being removed from the data set, see Figure 1.

Furthermore, in order to avoid erroneous observation of CEEs resulting from reflections from local surfaces, a quality filter pased on the summation method to obtain GHI was used (Gueymard and Myers, 2009). This filter calculates GHI from Equation (1) and compares it against the independently observed GHI. Observations where the ratio of calculated and observed GHI differ from 1.0 by more than 0.2 where excluded as they 
typically correspond to an error in the observation of at least 195 one irradiance component.

\subsection{Clear-Sky Model}

In addition to the measured irradiance data, a Clear-Sky ${ }_{199}$ Model (CSM) is used to characterize events that exceed the ${ }_{200}$ clear-sky ceiling. The clear-sky GHI is modeled using the $\mathrm{CSM}_{201}$ developed by Ineichen and Perez (2002), which requires Linke ${ }_{202}$ Turbidity as an input. Maps of monthly average Linke Turbidity ${ }_{203}$ developed by Remund et al. (2003) were used. These maps ac- ${ }_{204}$ count for seasonal changes of aerosol content in the atmosphere ${ }_{205}$ and their performance has been confirmed internally by Sandia ${ }_{206}$ National Laboratories (Reno et al., 2012). The CSM was calibrated against several clear days and a linear fit is used in order to ensure agreement between measured and modeled values.

\subsection{Statistical Analysis}

A statistical analysis was carried out in order to characterize the individual events that exceed the CSM into bounded ranges. Only GHI measurements that exceed the CSM by at least $5 \%$ and satisfy Equation (4) are considered potential CEEs. Potential CEEs are then grouped by the degree to which they exceed the CSM (5-10\%, 10-15\%, etc). Statistical analysis of these subsets of CEEs provides insight into the distribution and probability of GHI measurements as a function of the degree to which they exceed the CSM.

\subsection{Ramp Rate Analysis}

Analysis of the RRs uses the same set of GHI data from the statistical study with data not satisfying Equation (4) or violating the quality filter being removed. As was done in $\$ 3.3$, only events which exceed the CSM by a threshold of at least $5 \%$ are considered potential CEEs. However, rather than grouping the individual measurements by the range with which they exceed the clear-sky model $(5-10 \%, 10-15 \%$, etc), the present study employs a sliding lower bound which classifies coherent ramps by the amount with which their maximum value exceeds the CSM $(>5 \%,>10 \%$, etc), see Figure 5 .
In order to study the coherent CEE RRs, characteristic events must be located and quantified. This was accomplished through the identification of successive measurements which lie on opposite sides of the CSM. These points were then extrapolated to their respective local maxima and minima. This is illustrated in Figure 3 in which the ramps associated with CEEs, along with their local maxima and minima, have been identified in the Eppley PSP data from March 21, 2011 in Merced. It is important to note that while raw GHI data was used to locate potential CEEs (and the degree to which they exceed the CSM) the calculation of the RRs themselves ignores deterministic diurnal variations through the removal of the CSM values as described below,

$$
|R R|=\frac{\left(\mathrm{GHI}_{\max }-\mathrm{CSM}_{\max }\right)-\left(\mathrm{GHI}_{\min }-\mathrm{CSM}_{\min }\right)}{\Delta t} .
$$

\subsection{Wavelet Analysis}

Wavelet analysis allows for decomposition of non-periodic time-series into sets of orthogonal sub-signals representing fluctuations on specific time-scales, see for example Mallat (2009). For the purposes of the present study a wavelet similar to the top-hat wavelet employed in Lave et al. (2012, 2013) is also used here. The top-hat wavelet used here is centered at zero and is defined as,

$$
\psi(t)= \begin{cases}1 & -1 / 4<t<1 / 4 \\ -1 & -1 / 2<t<-1 / 4 \| 1 / 4<t<1 / 2 \\ 0 & \text { else }\end{cases}
$$

so that the dictionary of top-hat atoms can be written,

$$
\mathcal{D}=\left\{\psi_{\tau, 2^{j}}(t)=\frac{1}{\sqrt{2^{j+1}}} \psi\left(\frac{t-\tau}{2^{j}}\right)\right\}_{\tau \in \mathbb{R}, j \in \mathbb{Z}^{+}}
$$

where the wavelet has been offset by $\tau$ and scaled by $1 / \sqrt{2^{j+1}}$ in order to compensate for the total length of the wavelet. The corresponding wavelet transform is written

$$
\Psi f\left(\tau, 2^{j}\right)=\frac{1}{\sqrt{2^{j+1}}} \int_{-\infty}^{+\infty} f(t) \psi\left(\frac{t-\tau}{2^{j}}\right) d t
$$

Figure 4 shows the $\psi_{0,2^{2}}(t)$ top-hat wavelet centered at zero as well as two scaled, dilated, and translated versions of the 


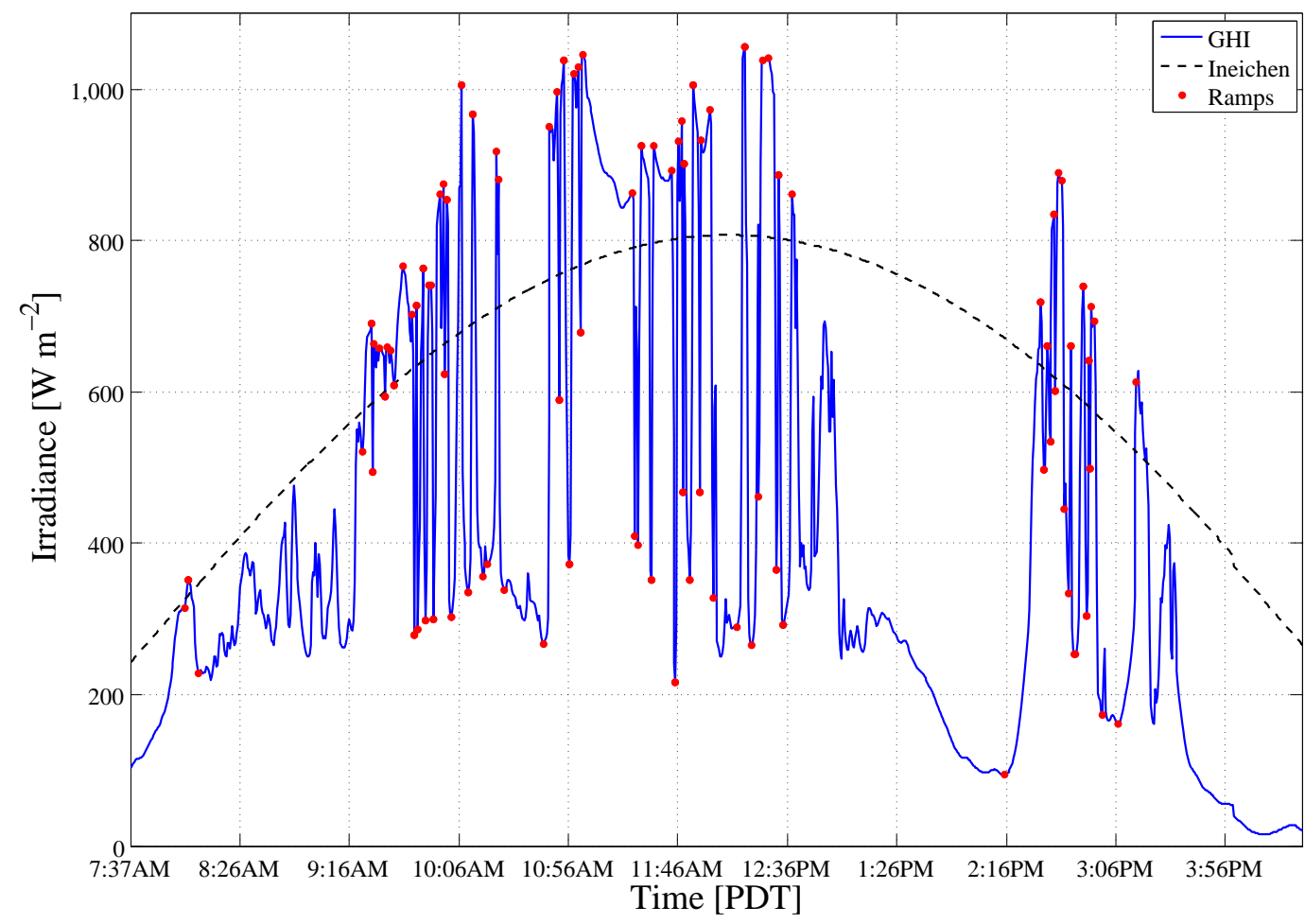

Figure 3: Ineichen clear-sky model as well as GHI data from the Eppley PSP located in Merced for the portion of March 21, 2011 satisfying Equation 4 The local maxima and minima of the coherent ramps associated with CEEs are highlighted in red. Coherent CEE ramps are defined as a set of monotonically increasing or decreasing irradiance observations whose maximum exceed the CSM by a specified threshold.

wavelet $\left\{\psi_{8,2^{3}}(t), \psi_{24,2^{4}}(t)\right\}$. These wavelets, which have a length of $2^{j}$, when applied to a stationary time-series of irradiance measurements would capture clear periods of length $2^{j-1}$ sur- $_{234}$ rounded by overcast periods or vice versa.

In order to determine the power contained in each of the time-series $\Psi f\left(\tau, 2^{j}\right)$, a wavelet periodogram $I_{j}(\tau)$ is calculated. In analogy to the Fourier periodogram as well as the work by Woyte et al. (2007) and Lave et al. (2012, 2013) the wavelet periodogram is defined as the square of the wavelet transform, normalized by the period of the wavelet,

$$
I_{j}(\tau)=\frac{\left[\Psi f\left(\tau, 2^{j}\right)\right]^{2}}{2^{j+1}}
$$

However, information regarding the variability from the wavelet periodograms is inconvenient to examine periods longer than a day. To remedy this, a fluctuation power index as described by Woyte et al. (2007) and employed in Lave et al. (2012) is used here to characterize the amount of power included in each time-scale. The fluctuation power index (fpi) is defined as,

$$
\operatorname{fpi}(j)=\frac{1}{T_{j}} \int_{0}^{T_{j}} \frac{\left[\Psi f\left(\tau, 2^{j}\right)\right]^{2}}{2^{j+1}} d \tau
$$

where $T_{j}$ is the total duration of the wavelet periodogram $I_{j}(\tau)$. 5 (36

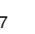
iscrete in nature. Therefore, discrete forms of equations (7-10) must be employed. The discrete forms are,

$$
\begin{gathered}
\mathcal{D}=\left\{\psi_{\tau, 2^{j}}(t)=\frac{1}{\sqrt{2^{j+1}}} \psi\left(\frac{t-\tau}{2^{j}}\right)\right\}_{\tau \in \mathbb{N}, j \in \mathbb{Z} \geq 2} \\
W f\left(\tau, 2^{j}\right)=\frac{\Delta t}{2 \sqrt{2^{j+1}}} \\
{\left[f(0) \psi\left(\frac{-\tau}{2^{j}}\right)+f(N) \psi\left(\frac{N-\tau}{2^{j}}\right)+2 \sum_{t=1}^{N} f(t) \psi\left(\frac{t-\tau}{2^{j}}\right)\right],} \\
I_{j}(\tau)=\frac{\left[W f\left(\tau, 2^{j}\right)\right]^{2}}{2^{j+1}},
\end{gathered}
$$

and

$$
\mathrm{fpi}(j)=\frac{\Delta t}{2 T_{j} \sqrt{2^{j+1}}}\left[I_{j}(0)+I_{j}\left(T_{j}\right)+2 \sum_{t=1}^{T_{j}-1} I_{j}(t)\right],
$$




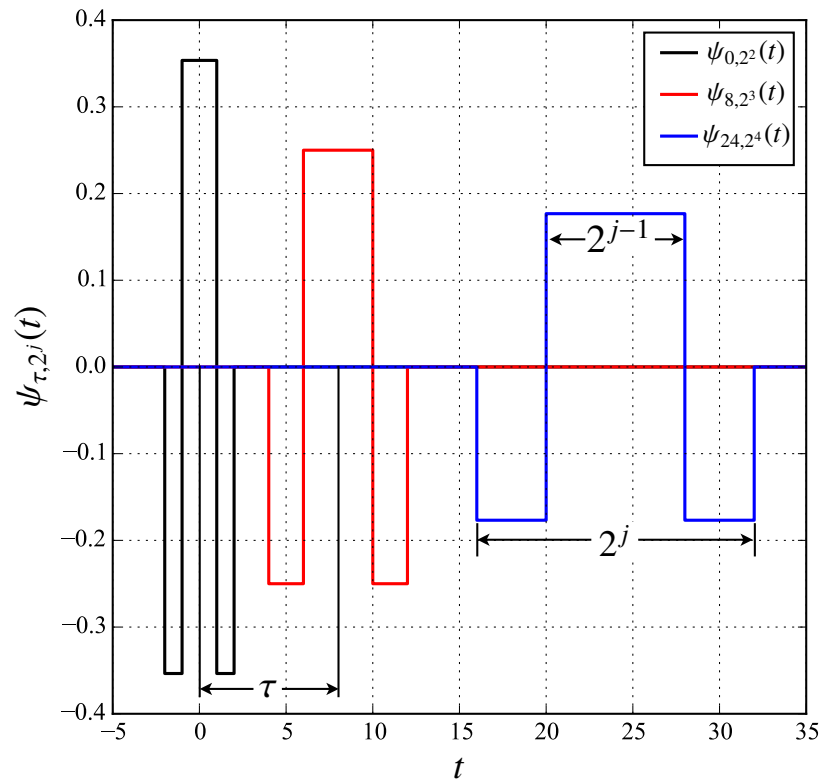

Figure 4: Illustration of the $\psi_{0,2^{2}}(t)$ top-hat wavelet centered at zero as well as two scaled, dilated and translated versions of the wavelet $\left\{\psi_{8,2^{3}}(t), \psi_{24,2^{4}}\right\}$.

respectively, where $\mathbb{N}$ represents integers between $2^{j-1}$ and $N{ }^{275}$ $2^{j-1}$.

\section{Results}

\subsection{Statistics}

A plot of the entire year of GHI data from Merced along 281 with suspected cloud enhancement events where the measured GHI exceeds the modeled clear-sky ceiling are shown in Figure ${ }^{282}$ 5 Allowing for errors in the measurement of GHI and modeling ${ }^{283}$ of clear-sky irradiance ceiling, potential enhancement events ${ }^{284}$ are associated with measured values which exceed the clear-285 sky model by at least $5 \%$. Our analysis shows that 10,228 of $^{286}$ the 262,281 data points, corresponding to approximately $4 \%$ of ${ }^{287}$ the data, qualify as potential CEEs. Multiplying the number ${ }^{288}$ of potential data points by 30 second time-steps corresponds to ${ }^{289}$ over 3.5 full-days of these events per year if considered sequen-290 tially. The surplus irradiance enhancements range from $18 \mathrm{~W}^{291}$ $\mathrm{m}^{-2}$ day $^{-} 1$ to $73 \mathrm{~W} \mathrm{~m}^{-2}$ day $^{-1}$. The maximum GHI in Merced ${ }^{292}$ was measured on May 8th, 2011 at 12:08p.m. $\left(\theta_{z}=20.5^{\circ}\right)$ with $^{293}$ a value of $1,365 \mathrm{~W} \mathrm{~m}^{-2}$, or approximately equal to the extrater-294 restrial beam irradiance $I_{0}$. This value is nearly $40 \%$ higher $^{295}$ than the modeled clear-sky ceiling suggesting substantial gains may result from these events.

Statistical results from the data in Merced as well as similar results for data sets collected in San Diego and Ewa Beach, are shown in Tables 2 and 3 respectively, all of which recorded maximum values of GHI $\geq I_{0}$. The maximum recorded GHI in San Diego of $1,396 \mathrm{~W} \mathrm{~m}^{-2}$, which is $42.7 \%$ higher than the modeled clear-sky ceiling, occurred on May 25, 2012 at 12:45p.m. $\left(\theta_{z}=17 \cdot 6^{\circ}\right)$. Similarly, a maximum recorded value of $1,380 \mathrm{~W} \mathrm{~m}^{-2}$, which is $32.95 \%$ higher than the modeled clear-sky ceiling, occurred on April 29, 2012 in Hawaii at 12:18p.m. $\left(\theta_{z}=7.2^{\circ}\right)$. It is beneficial to note that the magnitude, mean and deviation of these potential enhancement events across all three observatories do not vary greatly. In addition, cloud enhancements in Merced are seasonally dependent and are more likely to occur in the spring months. Moreover, there are very few potential cloud enhancement events during the summer months when the skies are relatively cloudless over Merced. Due to the difference in local climatology between Hawaii and California, there are less clear skies per year in Hawaii. Consequently, cloud enhancement events are more likely to occur and provide higher surplus local energy than the other two observatories during the same season, see Table 3 .

\subsection{Ramp Rate Probabilities}

Cumulative Distribution Functions (CDFs) of the previously defined coherent CEE RRs are shown in Figure 6 The CDF for the entire data set satisfying Equation (4) is also shown for reference and labeled as 'Day Values'. It should be noted that, as a result of varying climates, cloud induced RRs vary by location. Cloud induced RRs resulting in a change of at least $5 \%$ in $k_{G}$ for Merced, San Diego and Hawaii account for $6.86 \%, 15.6 \%$ and $44.3 \%$ of the data, respectively. It is clear from Figure 6 that the CEE RR distributions corresponding to increasing thresholds are quite similar. In addition, one can see that CEEs are associated with elevated RRs. Figure 6 also includes a horizontal line corresponding to $P=0.95$. The intersection of this line with the CDFs represent the RR magnitude which is exceeded 


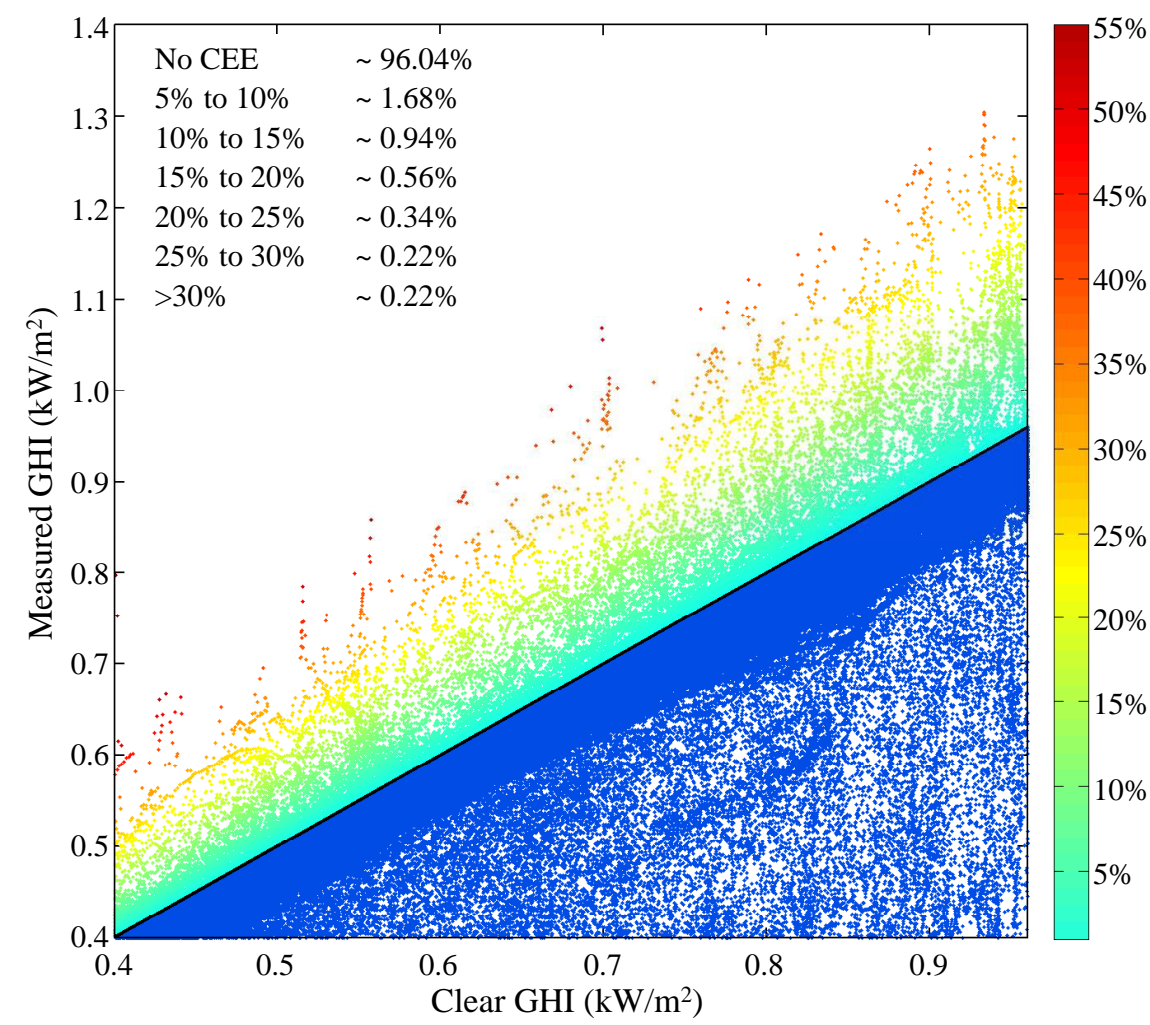

Figure 5: Plot of the entire year of GHI data from Merced as a function of the expected clear-sky value illustrating the set of potential CEEs.

\begin{tabular}{|l|cccccc|}
\hline Threshold & $\begin{array}{c}\text { Mean } \\
{\left[\mathrm{W} \mathrm{m}^{-2}\right]}\end{array}$ & $\begin{array}{c}\text { Standard } \\
\text { Deviation } \\
{\left[\mathrm{W} \mathrm{m}^{-2}\right]}\end{array}$ & $\begin{array}{c}\text { Occurrence } \\
{[-]}\end{array}$ & $\begin{array}{c}\text { Probability } \\
{[\%]}\end{array}$ & \multicolumn{2}{c|}{$\begin{array}{c}\text { Surplus } \\
\text { Energy }\end{array}$} \\
& {$\left[\mathrm{W} \mathrm{h} \mathrm{m}^{-2}\right]$} & {$\left[\mathrm{W} \mathrm{h} \mathrm{m}^{-2} \mathrm{day}^{-1}\right]$} \\
\hline $\mathbf{5 - 1 0 \%}$ & 53 & 16 & 4,238 & 1.62 & 1,850 & 4.06 \\
$\mathbf{1 0} \mathbf{- 1 5 \%}$ & 91 & 21 & 2,660 & 1.01 & 2,010 & 4.41 \\
$\mathbf{1 5}-\mathbf{2 0 \%}$ & 125 & 28 & 1,483 & 0.57 & 1,540 & 3.38 \\
$\mathbf{2 0} \mathbf{- 2 5 \%}$ & 157 & 37 & 890 & 0.34 & 1,170 & 2.57 \\
$\mathbf{2 5} \mathbf{- 3 0 \%}$ & 195 & 46 & 575 & 0.22 & 930 & 2.04 \\
$\geq \mathbf{3 0 \%}$ & 249 & 56 & 382 & 0.15 & 790 & 1.73 \\
\hline Total & 95 & 58 & 10,228 & 3.9 & 8,300 & 18.19 \\
\hline
\end{tabular}

Table 2: Statistical Results for CEE analysis for an entire time period (456 days) in Merced, CA.

\begin{tabular}{|l|ccccccc|}
\hline Location & Range & $\begin{array}{c}\text { Mean } \\
{[-]}\end{array}$ & $\begin{array}{c}\text { Standard } \\
\text { Deviation }\end{array}$ & $\begin{array}{c}\text { Probability } \\
{\left[\mathrm{W} \mathrm{m}^{-2}\right]}\end{array}$ & \multicolumn{2}{c}{$\begin{array}{c}\text { Surplus } \\
\text { Energy }\end{array}$} & $\begin{array}{c}\text { Fraction of } \\
\text { Total GHI }\end{array}$ \\
& {$\left[\mathrm{W} \mathrm{m}^{-2}\right]$} & {$[\%]$} & {$\left[\mathrm{W} \mathrm{h} \mathrm{m}^{-2}\right]$} & {$\left[\mathrm{W} \mathrm{h} \mathrm{m}^{-2} \mathrm{day}^{-1}\right]$} & {$[\%]$} \\
\hline Merced & Jan 29 - Jun 19, 2012 & 104 & 60 & 6.07 & 6,430 & 45.3 & $1.2 \%$ \\
San Diego & Jan 29 - Jun 17, 2012 & 92 & 51 & 5.59 & 5,370 & 38.4 & $1.0 \%$ \\
Hawaii & Jan 27 - Jun 17, 2012 & 101 & 52 & 10.71 & 10,350 & 72.9 & $1.3 \%$ \\
\hline
\end{tabular}

Table 3: Statistical results of CEEs at three observatories for the time period when all data from all sites was available (142 days). 
Table 4: Data used in the calculation of $P(R R)$ separated in to both up ramping events and down ramping events.

$5 \%$ of the time. This intersection occurs at approximately $76 \mathrm{~W}_{333}$ $\mathrm{m}^{-2} \min ^{-1}$ for the Day Values and an average value of $633 \mathrm{~W}_{334}$ $\mathrm{m}^{-2} \mathrm{~min}^{-1}$ for the CEE RR distributions. This suggests a significant increase in the probability of inflated RRs associated with $_{336}$ CEEs. The inset of Figure 6 shows the PDF of the $10 \% \mathrm{CEE}_{337}$ RR case as well as the PDF for the Day Values. The PDF is included to show the symmetry of the CEE RR distribution. This s39 $_{33}$ symmetry indicates that each ramp is associated with a corre- ${ }_{340}$ sponding ramp of similar magnitude and opposite sign. This is s $_{341}$ to be expected from the passing of a discrete and opaque cloud ${ }_{342}$ field. This behavior is also clear from a careful examination ${ }_{343}$ of Figure 3 and is summarized in Table 4 . Only the $10 \% \mathrm{PDF}_{344}$ case is shown for clarity, however it should be noted that the ${ }_{345}$ remaining distributions are quite similar.

\subsection{Wavelet Decomposition}

The wavelet transforms and periodograms from the clearsky index for Merced at each time-scale, $j=1$ to 7 for the entire year of data separated into days with and without CEEs of ${ }_{351}$ increasing thresholds were calculated. As mentioned in $\$ 3.5$, it ${ }_{352}$ is important to note that when the top-hat wavelet of time-scale ${ }_{353}$ $j$ is applied to a stationary time-series of irradiance measurements, it captures clear periods of length $2^{j-1}$ surrounded by overcast periods or vice versa. The wavelet transform and periodogram for March 21, 2011 over modes $j=1$ (60 seconds) ${ }_{357}$ to $j=7$ (about an hour) are shown in Figures 8 and 9 respectively. This day was chosen not only because it was used to il- ${ }_{359}$ lustrate the previous concepts but also because it has transitions ${ }_{360}$ between clear and cloudy periods at several time-scales which would be detected by the top-hat wavelet transform. Upon inspection, it is clear from Figure 8 that the wavelet transforms tend to be zero for times when no variability is present and increase in amplitude as fluctuations at the time-scale in question increase, as is to be expected. This is illustrated with clarity during the time period from approximately 1:00PM to 2:00PM, where only the $j=7$ mode has a non-zero amplitude while all other modes, i.e. $j=1-6$ are nearly zero. This is a result of the cloudy period that lasts on the order of an hour with very little variability on shorter time-scales. This result is even more evident upon inspection of the periodograms in Figure 9 where the periodogram is a non-negative number that increases in amplitude with the fluctuations at the time-scale in question.

As described in $\$ 3.5$ the average annual fluctuation power indices are plotted as a function of time-scale (mode) and CEE threshold in Figure 7(a). It is clear that days without CEEs tend to possess fluctuation power indices that are typically 5 to 15 times lower in magnitude, which is to be expected from the increased variability associated with the passing of cloud fields. However, regardless of the time-scale (mode) all of the fluctuation power indices for a particular threshold are maximum at the $j=6$ time-scale, suggesting that this is the dominant time-scale of cloud-fields/weather-systems that are in turn responsible for CEEs. To clarify, this mode corresponds to a clear period of 15 minutes surrounded by overcast periods or vice versa, which is in agreement with the results from Kassianov et al. (2004), which suggest that the typical decorrelation time for sky-cover is on the order of 15 minutes.

The ratio of the annual average fluctuation power indices of CEE to Non-CEE days are shown in Figure 7 (b) as a function of CEE threshold. This is performed in order to examine the impact of CEEs at each time-scale; that is, at which time-scale do the CEEs have the most impact. Unlike the results in Figure 7(a), which show a peak at the $j=6$ mode associated with approximately 30 minutes, the ratios show a peak at the $j=3$ mode which is associated with a time-scale of approximately 4 minutes. This suggests that while the clouds that are responsi- 


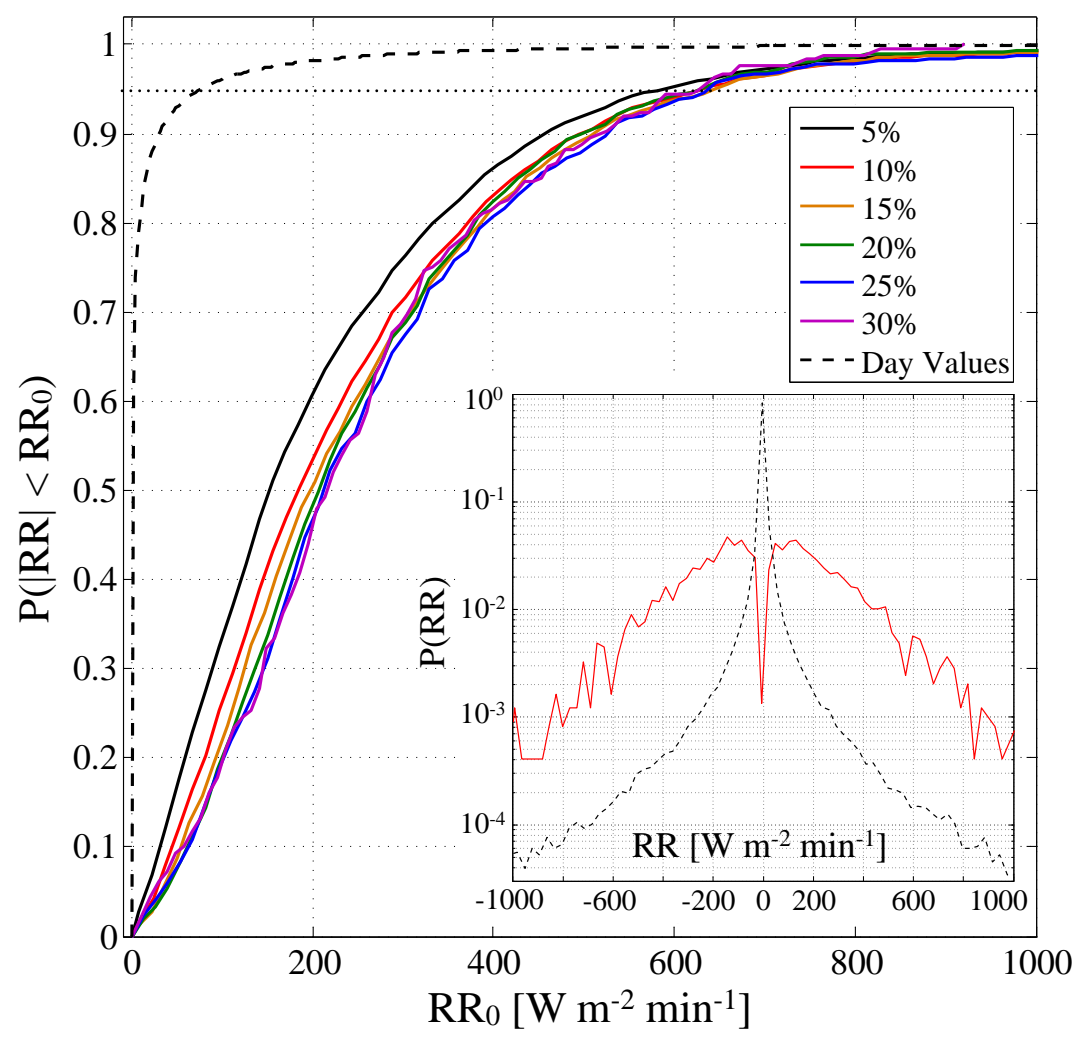

Figure 6: Cumulative Distribution Functions (CDFs) of the coherent Ramp Rates (RRs) whose maximum values exceed the CSM by a increasing threshold and are associated with Cloud Enhancement Events (CEEs). The CDF of the entire year of data that satisfy Equation (4) is also shown for comparison and labeled as 'Day Values'. Intersection of the $\mathrm{P}=0.95$ line and the Day Values CDF occurs at $76 \mathrm{~W} \mathrm{~m}^{-2} \mathrm{~min}^{-1}$ and an average value of $633 \mathrm{~W} \mathrm{~m}{ }^{-2} \mathrm{~min}^{-1}$ for the CEE RRs. Inset: Probability Distribution Function (PDF) of the Day Values as well the 10\% threshold distribution showing the symmetry of RR associated with the passing of opaque clouds as well as the increased probability of elevated RRs. 
ble for creating CEEs tend to introduce variability on the order 398 30 minutes, the CEEs themselves tend to operate on time-scales ${ }_{399}$ about one eighth as long ( 4 minutes).

\section{Conclusions}

This study investigates the observational frequency of $\mathrm{CEEs}_{402}$ and the associated coherent RRs. A statistical analysis showed ${ }_{403}$ that nearly $4 \%$ of the observations at Merced qualify as potential CEEs corresponding to nearly 3.5 days of CEEs per year. The increases above the predicted clear-sky ceiling for these CEEs ranged from 10 to $520 \mathrm{~W} \mathrm{~m}^{-2}$ and maximum GHI values on the order of $1,300 \mathrm{~W} \mathrm{~m}^{-2}$ were observed at all three observatories. The maximum CEE was observed in San Diego on the 25th of May, 2012, with a value of nearly 1,400 $\mathrm{W} \mathrm{m}^{-2}$, which was $43 \%$ higher than the modeled clear-sky ceiling.

Cumulative Distribution Functions (CDFs) show that CEEs are associated with elevated RRs. For days with no CEEs RRs greater than $76 \mathrm{~W} \mathrm{~m}^{-2} \mathrm{~min}^{-1}$ occur only $5 \%$ of the time. On the other hand, for days containing CEEs RRs exceeding 633 $\mathrm{W} \mathrm{m} \mathrm{m}^{-2} \mathrm{~min}^{-1}$ occur $5 \%$ of the time. In addition, Probability Distribution Functions (PDFs) were used to illustrate the symmetry of the CEE RR distributions, which suggests that each CEE RR is associated with a ramp of similar magnitude and opposite sign.

Finally, wavelet decomposition coupled with fluctuation power index analysis shed light on the time-scales on which cloud induced variability and CEEs operate. An examination of the magnitude of the fluctuation power indices showed that regardless of the presence of CEEs, cloud induced variability tents to have a maximum fluctuation power index at the $30 \mathrm{~min}$ timescale. However, upon inspection of the ratios of the fluctuation power indices for both CEE and non-CEE at each time-scale showed a maximum increase at the 4 minute time-scale, regardless of CEE threshold. In other words, this result suggest that while clouds tend to induce variability most strongly at the 30 minute time-scale, they have the potential to cause CEEs that induce variability on time-scales of several minutes. This analysis clearly demonstrates that CEEs are an indicator for periods of high variability and therefore provide useful information for solar forecasting and integration.

\section{Acknowledgements}

The authors gratefully acknowledge funding from the California Energy Commission (CEC) Project EPC-14-008 and DOE SUNRISE project number 0865-1517. 


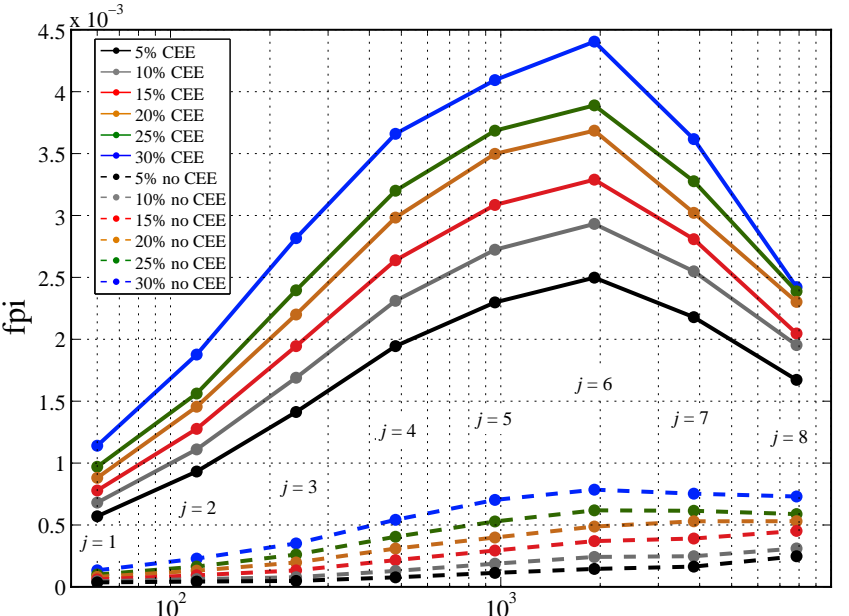

Timescale [s]

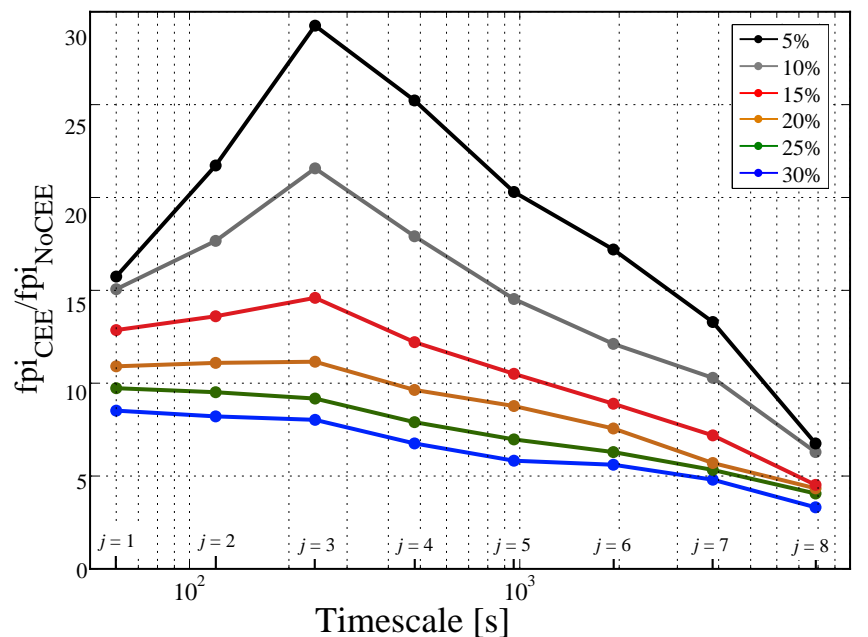

Figure 7: (a) Average annual fluctuation power indices as a function of time-scale and CEE threshold. Days without CEEs tend to possess fluctuation power indices that are typically 5 to 15 times lower in magnitude. Regardless of the time-scale (mode) all of the fluctuation power indices for a particular threshold are maximum at a time-scale of approximately 30 minutes $(j=7)$, suggesting that this is the dominant time-scale of clouds that are in turn responsible for CEEs. (b) Ratios of annual average fluctuation power indices of CEE to non-CEE days as a function of CEE threshold. Unlike (a), which show a peak at the $j=7$ mode associated with approximately 30 minutes, the ratios show a peak at the $j=4$ mode which is associated with a time-scale of approximately 4 minutes, suggesting that while the clouds that are responsible for creating CEEs tend to introduce variability on the order 30 minutes, the CEEs themselves tend to operate on time-scales about one eighth as long ( 4 minutes) 

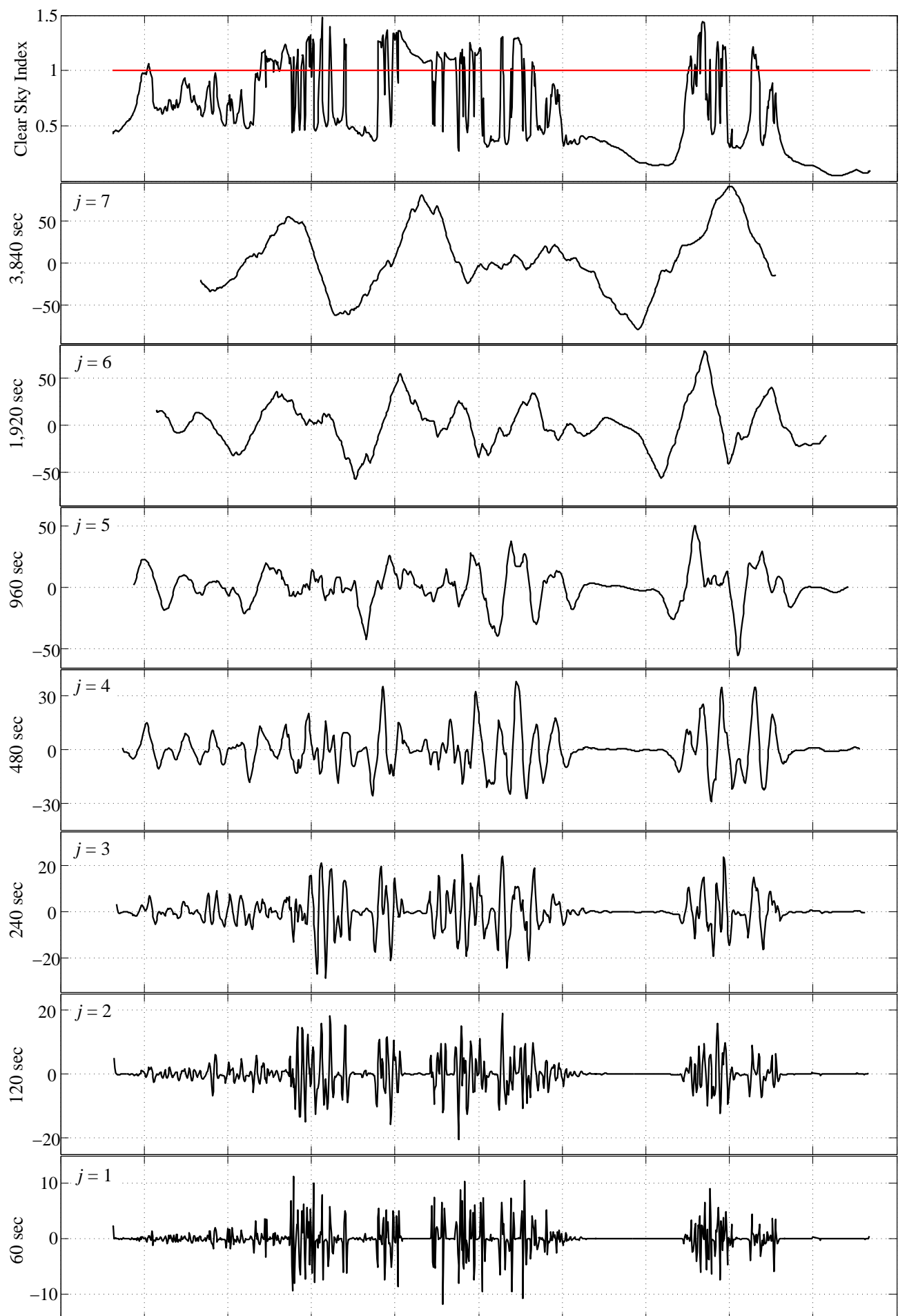

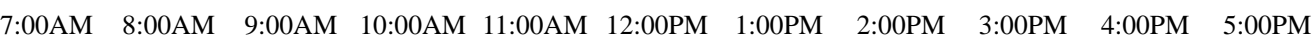

Figure 8: The wavelet transforms from the clear-sky index for Merced at each time-scale, $j=2-8$ for the entire day of March 21, 2011 satisfying Equation (4). 

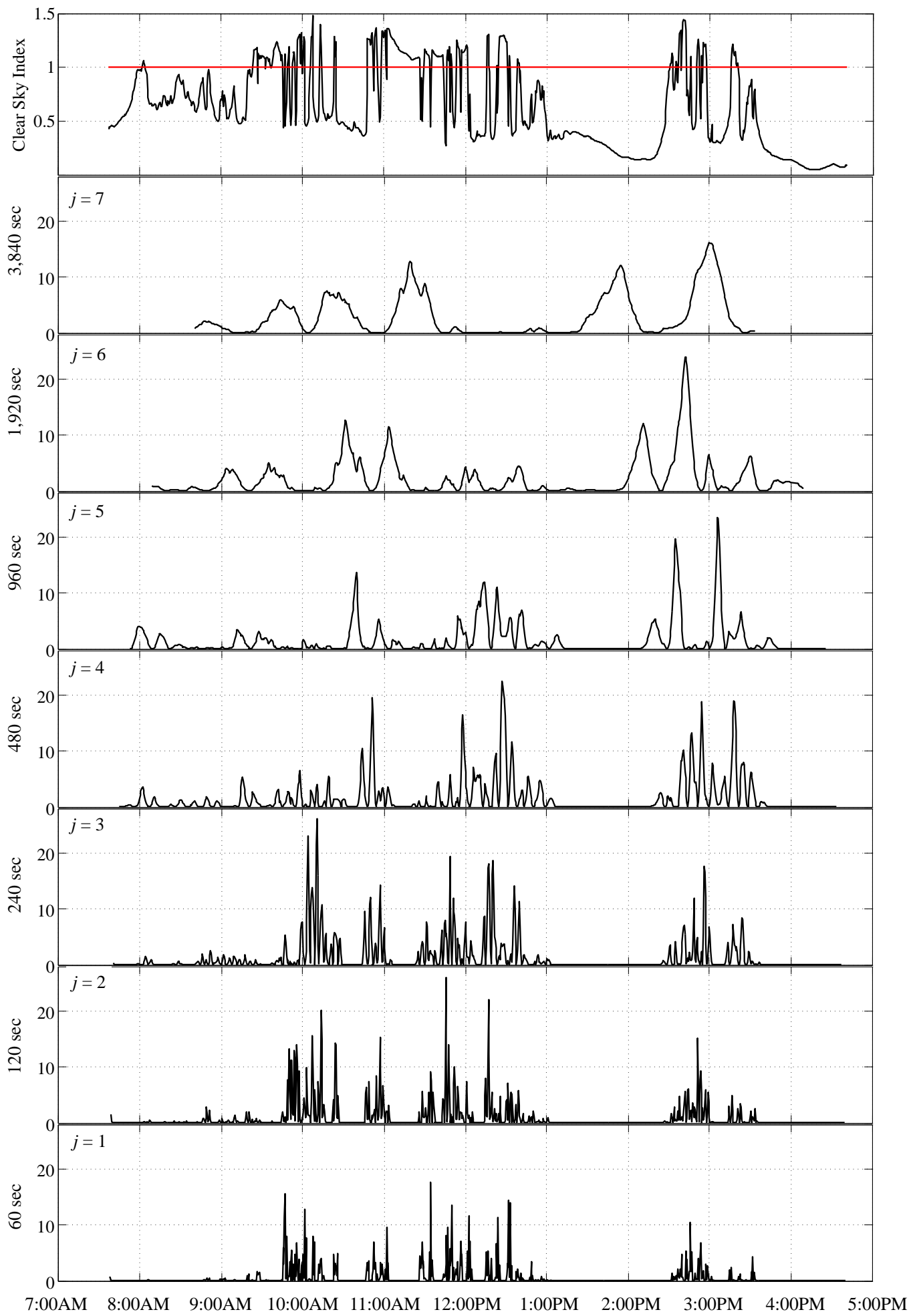

Figure 9: The wavelet periodogram from the clear-sky index for Merced at each time-scale, $j=2-8$ for the entire day of March 21, 2011 satisfying Equation 4 . 
Almeida, M. P., Zilles, R., Lorenzo, L., 2014. Extreme overirradiance events in Sao Paulo, Brazil. Sol. Energy 110, 168-73.

Berg, L. K., Kassianov, E. I., Long, C. N., Mills, D. L., 2011. Surface summertime radiative forcing by shallow cumuli at the Atmospheric Radiation Measurement Southern Great Plains site. J. Geophys. Res. 116 (D01202), $1-13$.

Cede, A., Blumthaler, M., Luccini, E., Piacentini, R. D., Nuñez, L., 2002. Effects of clouds on erythemal and total irradiance as derived from data of the Argentine Network. Geophys. Res. Lett. 29 (24), 1-4.

Emck, P., Richter, M., November 2008. An Upper Threshold of Enhanced Global Shortwave Irradiance in the Troposphere Derived from Field Measurements in Tropical Mountains. J. Appl. Meteorol. Clim. 47 (11), 28282845 .

Franceschini, G. A., 1968. The Influence of Clouds on Solar Radiation at Sea. Deutsche Hydrografische Zeitschrift 21 (4), 162-68.

Goody, R. M., Yung, Y. L., 1995. Atmospheric Radiation: Theoretical Basis, Second Edition. NewYork, Oxford University Press.

Gueymard, C. A., Myers, D. R., 2009. Evaluation of conventional and highperformance routine solar radiation measurements for improved solar resource, climatological trends, and radiative modeling. Sol. Energy 83 (2), 171-185.

Haar, A., 1911. Zur Theorie der orthogonalen Funktionensysteme. Math. Ann $71(1), 38-53$

Hazewinkel, M., 2001. Daubechies Wavelets, Encyclopedia of Mathematics, Springer Science \& Business Media.

Ineichen, P., Perez, R., 2002. A new airmass independent formulation for the Linke turbidity coefficient. Sol. Energy 73 (9), 151-157.

Inman, R. H., Pedro, H. T. C., Coimbra, C. F. M., 2013. Solar forecasting methods for renewable energy integration. Prog. Energ. Combust. 39 (6), 535576.

Kassianov, E., Long, C. N., Ovtchinnikov, M., 2004. Cloud Sky Cover versus Cloud Fraction: Whole-Sky Simulations and Observations. J. Appl. Meteorol. 44 (1): 86-98

Kawasaki, N., Oozeki, T., Otani, K., Kurokawa, K., 2006. An evaluation method of the fluctuation characteristics of photovoltaic systems by using frequency analysis. Sol. Energ. Mat. Sol. C. 90 (18-19), 3356-3363. dence and climate significance. Geophys. Res. Lett. 38 (L01706), 1-7. , Kleissl, J., Arias-Castro, E., 2012. High-frequency irradiance fluctuations and geographic smoothing. Sol. Energy 86 (8), 2190-2199.

Lave, M., Kleissl, J., Stein, J. S., 2013. A Wavelet-Based Variability Model (WVM) for Solar PV Power Plants. IEEE Transactions on Sustainable Energy 4 (2), 1-9.

Luoma, J., Kleissl, J., Murray, K., 2012. Optimal inverter considering cloud enhancement. Sol. Energy 86 (1), 421-429.

Mallat, S., 2009. A Wavelet Tour of Signal Processing: The Sparse Way, Third
Edition. Academic Press.

McCormick, P. G., Suehrcke, H., 1990. Cloud-reflected radiation. Nature 345, 773.

Peled, A., Appelbaum, J., 2013. Evaluation of solar radiation properties by statistical tools and wavelet analysis. Renew. Energ. 59 (11), 30-38.

Perpiñán, O., Lorenzo, E., 2011. Analysis and synthesis of the variability of irradiance and PV power time series with the wavelet transform. Sol. Energy 85 (1),188-197.

Perpiñán, O., Marcos, J., Lorenzo, E., 2013. Electrical power fluctuations in a network of DC/AC inverters in a large PV plant: Relationship between correlation, distance and time scale. Sol. Energy 88 (2), 227-241.

Pfister, G., McKenzie, R. L., Liley, J. B., Thomas, A., Forgan, B. W., Long, C. N., 2003. Cloud Coverage Based on All-Sky Imaging and Its Impact on Surface Solar Irradiance. J. Appl. Mereorol. Clim. 42 (10), 1421-1434.

Remund, J., Wald, L., Lefèvre, M., Ranchin, T., Page, P., 2003. Worldwide Linke Turbidity Information. In: Proceedings of ISES Solar World Congress. Götenborg, Sweden.

Reno, M. J., Hansen, C. W., Stein, J. S., 2012. Global Horizontal Irradiance Clear Sky Models: Implementation and Analysis. Tech. Rep. SAND20122389, Sandia National Laboratories.

Schade, N. H., Macke, A., Sandmann, H., Stick, C., 2007. Enhanced solar global irradiance during cloudy sky conditions. Meteorol. Z. 16 (3), $295-$ 303 .

Segal, M., Davis, J., 1992. The Impact of Deep Cumulus Reflection on the Ground-Level Global Irradiance. J. Appl. Meteorol. Clim. 31 (2), 217-222.

Tapakis, R., Charalambides, A. G., 2014. Enhanced values of global irradiance due to the presence of clouds in Eastern Mediterranean. Renew. Energ. $62(2), 459-467$

Wen, G., Cahalan, R. F., Tsay, S., Oreopoulos, L., 2001. Impact of cumulus cloud spacing on Landsat atmospheric correction and aerosol retrieval. $J$. Geophys. Res. 106 (D11), 1-12.

Woyte, A., Belmans, R., Nijs, J., 2007. Fluctuations in instantaneous clearness index: Analysis and statistics. Sol. Energy 81 (2), 195-206.

Wyser, K., O’Hirok, W., Gautier, C., Jones, C., 2002. Remote sensing of surface solar irradiance with corrections for 3-D cloud effects. Remote Sens. Environ. 80 (2), 272-84.

Yordanov, G. H., Midtgrd, O. M., Saetre, T. O., Nielsen, H. K., Norum, L. E., 2013a. Overirradiance (cloud enhancement) events at high latitudes. IEEE Journal of Photovoltaics 3 (1), 271-77.

Yordanov, G. H., Saetre, T. O., Midtgard, O. M., 2013b. 100-millisecond resolution for accurate overirradiance measurements. IEEE Journal of Photovoltaics 3 (4), 1354-60. 